\title{
Embracing the organics world
}

\author{
Mature fabrication technologies and a healthy research and development environment promise a \\ glowing future for organic semiconductors.
}

Displays for smartphones, coloured light sources, portable solar cells and curved television screens are just but a few examples of the increasing presence of organic electronics in our daily lives. Although semiconducting organic molecules and polymers cannot yet compete with their inorganic counterparts in terms of chargetransport performance and industrial development, they hold key unrivalled advantages: reduced production costs, versatility of synthesis processes, and compatibility with a vast range of substrates such as transparent glass and flexible polymeric materials. In fact, several hightech companies have significantly invested in cheap and high-performance organicelectronic devices, a billion-dollar market that is expected to grow rapidly ${ }^{1}$. Over the years, this journal has contributed to reporting the technologically successful stories of organic light-emitting diodes (OLEDs), photovoltaics and thin-film transistors (TFT), as well as pioneering explorations in organic spintronics, organic sensors and stretchable electronics $^{2-5}$. In this issue, six research papers and two News \& Views articles highlight the latest advances and challenges in these fields.

OLED-based displays and lighting systems are undoubtedly the leading products driving the market of organic semiconductors. However, as noted by Chris Groves on page 597, the development of the multilayered structure of white-emitting OLEDs still relies on trial and error rather than rational theoretical design. On page 652, Peter Bobbert and colleagues report a three-dimensional model that simulates, with molecular precision, the behaviour of charges and excitons in these devices. Rational approaches such as this could aid - or eventually replace - the Edisonian optimization of OLEDs and similar multilayered architectures (such as tandem and multi-junction solar cells), and thus accelerate their penetration into the marketplace.

In terms of technological transfer, organic transistors lag a step behind OLEDs. For example, randomly deposited layers of molecules or polymers that are characterized by a locally varying conductivity typically lead to poor process-to-process and deviceto-device reproducibility. Good control over the molecular orientation and crystallinity of organic films is therefore desirable.
However, as Antonio Facchetti argues on page 598 , this is particularly challenging for solution-based thin-film deposition, so far the cheapest and most versatile technology for large-scale production. Two approaches described in this issue address the problem of obtaining long-range ordered films from different perspectives: molecular design and fluid-flow control. On page 659, Jinsang Kim and co-workers show that, by engineering the molecular structure of conjugated polymers, these tend to crystallize with exceptional regularity when deposited from solution and spread on a substrate. On page 665 , Zhenan Bao and collaborators describe the use of a microstructured blade that 'combs' an organic solution during evaporation to guide the uniform distribution and nucleation of the semiconducting molecules dispersed in the solvent. They achieved crystalline molecular packing in centimetre-long domains, and fabricated TFT arrays with a remarkably higher mobility and reduced device-todevice variation. In a complementary approach, Michael Chabinyc and colleagues demonstrate on page 628 that commercial additives - a widely used strategy in the crystallization of bulk polymers - promote the fast nucleation of crystallites in organic semiconducting films. Indeed, decreasing evaporation times can be beneficial for electronic devices fabricated on flexible substrates (as these have limited resistance to thermal stress). This should capture the attention of those companies that are leveraging flexibility and transparency - the most advertised characteristics of organic transistors - to compete with the more mature inorganic devices.

The enhanced biocompatibility of organic materials has also led to biomedical applications, in particular in neuron interfacing. Recent publications have demonstrated that charges flowing in organic channels can interact with the electric field generated by action potentials $s^{6,7}$. On page 672, Michele Muccini and co-authors show that transparent organic transistors can simultaneously sense and stimulate the electrical activity of cultured mammalian neurons. Although there is a long way to go to reach the single-neuron precision of siliconbased neuronal transducers, transparent and mechanically compliant tools based on biocompatible organic materials could

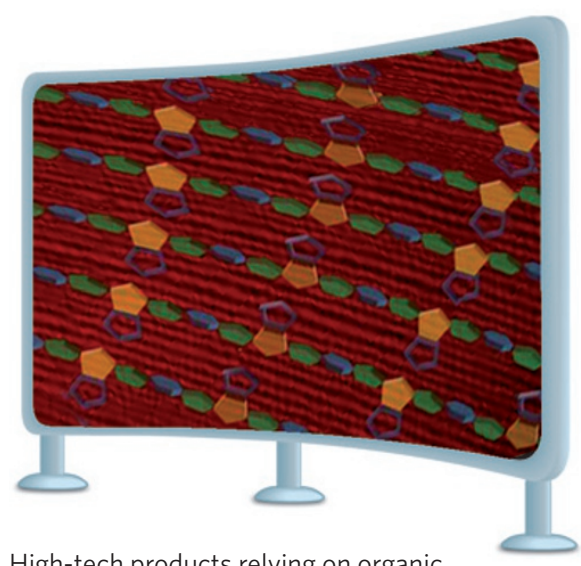

High-tech products relying on organic semiconductors, such as curved

television screens, demonstrate the industrial maturity of organic electronics. Image on screen courtesy of Bong-Gi Kim and Jinsang Kim.

open unprecedented possibilities for neurophysiology.

In spintronics, Kazuya Ando and collaborators report on page 622 that efficient spin-charge conversion can also be achieved in organic materials by using ferromagnetic insulators to inject spin current in a highly conductive conjugated polymer. Small voltage signals are generated between gold contacts deposited on the organic layer, which is a proof of the inverse spin Hall effect detected in materials characterized by a long spin lifetime. Organic spintronic devices are far from reaching industrial maturity, yet significant progress is still being made ${ }^{8}$.

Recent improvements in organic devices are largely due to new design tools and processing strategies, and we hope that the fundamental, methodological and technical advances reported in this issue will contribute to broadening the range of applications involving polymers and organic molecules alongside (or in place of) inorganic semiconductors. Certainly, for organic electronics the world is no longer small.

\footnotetext{
References

1. Das, R. \& Harrop, P. Printed, Organic \& Flexible Electronics: Forecasts, Players \& Opportunities 2013-2023 (2013); available via http://go.nature.com/BxrL2J

2. Gelinck, G. H. et al. Nature Mater. 3, 106-110 (2004).

3. Li, G. et al. Nature Mater. 4, 864-868 (2005).

4. Sekitani, T. et al. Nature Mater. 8, 494-499 (2009)

5. Sun, Y. et al. Nature Mater. 11, 44-48 (2012).

6. Cramer, T. et al. Phys. Chem. Chem. Phys. 15, 3897-3905 (2013).

7. Khodagholy, D. et al. Nature Commun. 4, 1575 (2013).

8. Nature Mater. (Organic Spintronics web focus; 2009); available via http://go.nature.com/ybsGbk
} 